(Aus der deutsohen Univ.-Augenklinik zu Prag. [Vorstand: Prof. Dr. A. Elschnig.])

\title{
Schwund markhaltiger Nervenfasern in der Netzhaut nach Embolie der Art. centralis retinae.
}

\author{
Von \\ Dr. Rudolf Bachmann, \\ tixternarzt der Augenklinik Prag, dz. Klin. Assistent Bern.
}

Mit 3 Textabbildungen.

Das Verschwinden markhaltiger Nervenfasern in der Netzhaut wurde erstmals von Wagen mann $n^{1}$ ) im Verlaufe einer genuinen Sehnervenatrophie bei Tabes dorsalis beobachtet. Die zweite Mitteilung einer analogen Beobachtung stammt von Sachsalber ${ }^{2}$ ) bei einer in Atrophie übergegangenen Stauungspapille durch Hirntumor.

Wie Leber ${ }^{3}$ ) berichtet, hat überdies Nettleship einmal bei einer Papillitis einen peripheren Herd markhaltiger Nervenfasern, Frost ein Markbüschel an der Papille durch Glaukom verschwinden gesehen ohne genaue Beobachtung des Verlaufes.

Diesen spärlichen Fällen reiht sich nun folgende Beobachtung an.

K., Emil, 28 Jahre, aufgenommen am 22. I. 1920. Vor 8 Tagen plötzlich am rechten Auge innerhalb weniger Minuten erblindet. Im Kriegsdienst 1918 Malaria durchgemacht, seit September 1918 geheilt.

Groß, kräftig, gut genährt, leichte Struma. Abgeheilter Prozeß in der rechten Lungenspitze. Kom pensierte Insuffizienz der Mitralis. Geringer chronis cher Milzt u mor. Positive Tuberkulin-Stichreaktion, im übrigen alles normal; Wassermann negativ.

22. I. 1920: Rechtes Auge in leichter Divergenzstellung, Pupille rund, $6 \mathrm{~mm}$ gegen $4^{1 / 2} \mathrm{~mm}$ links, auf Lichteinfall nicht reagierend.

An die Papille anschließ3end prachtvolle markhaltige Nervenfasern, oben ein kleineres Bündel, das sektorartig zwischen $1 / 212$ und $1 / 22^{\mathrm{b}}$ den Papillenrand überdeckt und ganz wenig auf die Netzhaut übergreift. Die temporale Begrenzung dieses Bündels ist durch den aufsteigenden, leicht geschwungenen Ast der Zentralvene gebildet, während die nasale Grenze beinahe ein zilioretinales Gefäß erreicht, welches in einiger Entfernung vom Papillenrand aus dem braunrot gefärbten Papillengewebe auftaucht. Ebenso ist ungefähr das untere Segmentdrittel der Papille durch eine mächtige Entwicklung markhaltiger Nervenfasern von etwa Papillenausdehnung eingenommen. Die sich überkreuzenden unteren

1) Wagenmann, Graefes Arch. f. Ophthalmol. 40 (4), S. 256.

2) Sachs alber, Zeitschr. f. Augenheilk. 13, Erg.-Heft, S. 739.

3) Leber, Die Krankheiten der Netzhaut. Graefe-Saemisch Handb. 2. Aufl., 2. Hälfte, Bd. VII, S. 2036. 
Retinagefäße teilen dieses Bündel in eine größere nasale und kleinere temporale Partie und werden selbst vom peripheren Teil desselben verdeckt, so namentlich die Teilungsstelle der Vene.

Die Papille ist nasal durch einen Pigmentring deutlich begrenzt, temporal geht sie vollständig unscharf begrenzt in eine schmutziggraue bis weiße Trübung der Netzhaut über, die in einer Ausdehnung von 5 Papillendurchmesser horizontal und etwa 3 Papillendurchmesser vertikal, dann unscharf begrenzt, gegen die Peripherie zu allmählich in die normale Netzhaut ïbergeht. Die Foveagegend ist als dunkelgrauroter Fleck markiert, über den die getrübte, veränderte Netzhaut deutlich prominiert.

Die Arterien sind sowohl an der Papille wie in der N'tzhaut gefüllt, nirgends ist ein Embolus sichtbar. Die Venen sind erweitert, nasal oben in 3 Papillendurchmesser-Entfernung bedeckt eine größere Blutung Gefäße und Netzhaut. Auch auf ziemlichen Druck während des Ophthalmoskopierens sind weder Venen noch Arterien zum Kollaps zu bringen. $\mathrm{S}=$ Fingerzählen exzentrisch außen in $20 \mathrm{~cm}$. Linkes Auge vollständig normal.

In den nächsten Wochen bildet sich die ischämische Trübung unter Auftreten mehrerer kleiner rundlicher Blutungen in der Maculagegend zurück, während die größere Hämorrhagie nasal oben restlos resorbiert wird. Eine Veränderung der markhaltigen Nervenfasern wird bereits anfangs Februar wahrgenommen; sie besteht in einer leichten Auflockerung in feinst silberglänzende Fleckchen.

16. III. 1920: Das Papillengewebe ist bereits blasser als beim Eintritt, jedoch noch deutlich gelbrot. Einige sehr feine, gewundene Gefäßchen werden im Papillengewebe sowie im Bereich der unteren markhaltigen Nervenfasern sichtbar. (Abb. 1.)

Das obere markhaltige Büschel ist flaumartig, aber noch wenig verändert den Verlauf der kleinen, bei $1^{\mathrm{h}}$ zutage tretenden Vene sieht man wie einen dünnen roten Faden durchschimmern. Ebenso ist die temporale untere Partie, die insbesondere die unteren Gefäße wie mit feinen Schneeflöckchen deckt und als zartes Schleierchen die Teilung der unteren Arterie begleitet, noch kraum verändert. Dagegen ist im nasalen unteren Quadranten die Struktur der Fasern erheblich anders geworden: die sonst homogene Anordnung ist hier in mehr und minder hellglänzende, in ihrem Verlauf mehrfach unterbrochene Facern aufgelöst und macht den Eindruck zerschlissener weißer Seide, unter der die Unterlage durchschimmert. Am Papillenrand ist die Veränderung am weitesten vorgeschritten.

In der Foveagegend ist ein graubrauner Herd, daneben ein kleines rundes, hellgelb-weißes Fleckchen. Das ganze Gebiet der ehemals ischämischen Trübung, namentlich unterhalb der Macula ist mit zahllosen kleinsten Glitzerpünktchen übersät. Zahlreiche obliterierte GefäBteilungen imponieren als gelbweiße gegabelte feine Linien, die von oben und von der Papille her der Fovea zustreben; zum Teil sind sie noch als Fortsetzung bluthaltiger Gefäße zu verfolgen. Dazwischen finden sich dunklere Flecke als Reste von Blutungen.

28. IV. 1920: Die Papille is t sehr bla B, mit feinen Gefäßschlingen. Die Papillengefäße sind leicht zum Pulsieren und Kollaps zu bringen, die Arterien enger als die Venen.

Markhaltige Nervenfasern: Von der nasal unten zuerst vom Zerfall ergriffenen Partie sind nur mehr spärliche, segmentierte Reste mit einzelnen feinen Pigmenteinlagerungen vorhanden. (Abb. 2.)

Das Gros des unteren Bündels, insbesondere die nasal von der unteren Vene gelegene Stelle am Papillenrand sowie über und oberhalb der Venenteilung, die bereits gut sichtbar geworden ist, ist stark rarefiziert. Desgleichen machte der Zerfall der temporalen Zone, auch hier wieder zuerst über dem Papillenrand und über 


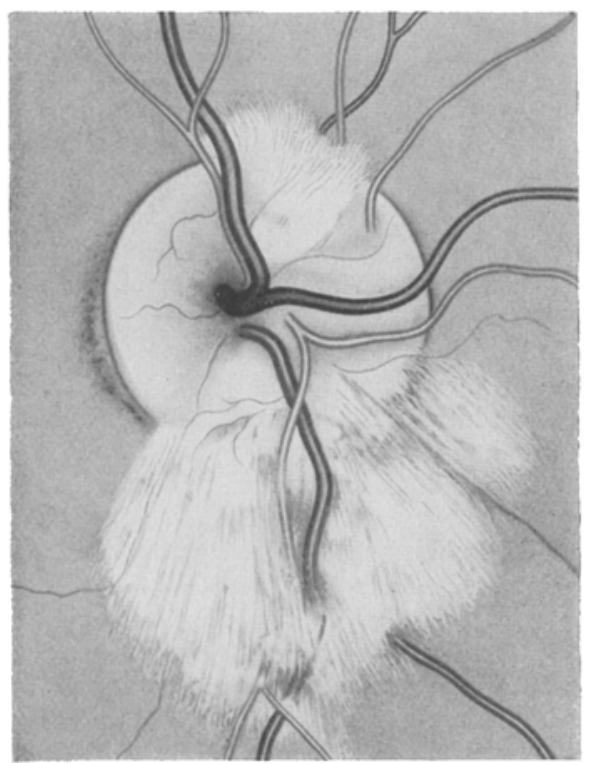

Abb. 1. Yor Beginn des Nervenfaserschwundes.

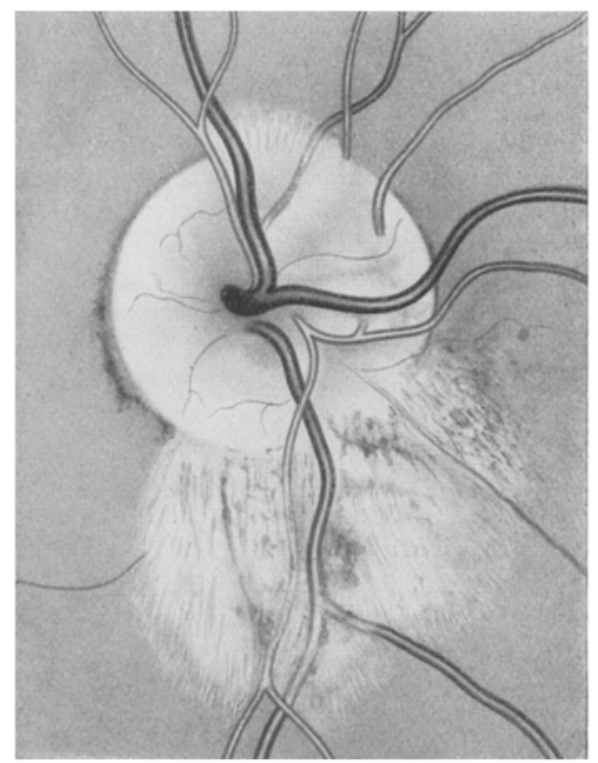

Abb. 2. Beginn des Nervenfaserschwundes.

der Arterie, große Fortschritte-es sieht aus, wie wenn von einer Lage weißer Watte nur mehr ein verschieden dichter, zerzupfter Schleier übriggeblieben wäre. Die Begrenzung des Bündels ist nicht mehr scharf, sondern ein ganz all mählicher. Übergang in den Fundus. Das obere Bündel hält sich am längsten, doch ist an dem breiter gewordenen Band der bedeckten Vene ein Fortschritt des Zerfalls zu bemerken. In der Macula bilden sich kleine Pigmentherdchen, während sich die Blutungsreste resorbieren und die Zahl der Glitzerpünktchen abnimmt, Die Obliteration der Gefäßchen geht nieht weiter.

3. V. 1920: Pat. wird entlassen und erscheint am 13. IV. 1921 zur Kontrolle wieder. (Abb. 3, gez. von Dr. Koch.)

Rechtes Auge. Fundus: Pa pille fast sch neewei $\beta$ und nasal oben rötlicher Schimmer vom Pigmentring allenthalben eingesäumt, Grenze nicht scharf. Von der nasalen unteren Papillenarterie geht ein kleiner Ast ab, der unter der Vene durchgeht und undeutlich sichtbar in die scheinbar zilioretinale Arterie umbiegt. Die Arterien sonst im großen unverändert, eng. Unterhalb der Papille weißliche Fleckchen, meistens fast kreisförmig (unter den früheren markhaltigen Nervenfasem). In der Mitte dieser weißlichen Herde an 2 Stellen kleinste schwarze Pünkt. chen. Um die Papille herum Pig. mentepithel unverändert deutlich zu sehen.

Ubriger Fundus gelblichrot gefärbt. Die oberhalb und unter. halb der Macula befindlichen gelbgläruenden Streifen (obliterierte GefäBe) wie früher. In der $\mathrm{Ma}$ culagegend schwarze, zu Gruppen zusammengeschmolzene Herdchen in einem ca. 1/2 Papillenausdehnung hohen, 1/a Papillenausdehnung breiten, ovalen Bezirke. An seinen. Grenzen zahlreiche Glitzerpünktahen. 
Paramacular, besonders unten, auch peripapillär zahlreiche feinste, sandartige Pigmentfleckchen.

$\mathrm{S}=$ Handbewegungen exzentrisch außen unten.

Nach Mitteilung der Krankengeschichte, die mit Rücksicht auf das bisher noch nicht genauer beobachtete ophthalmoskopische Bild des Schwundes markhaltiger Nervenfasern etwas ausführlicher wiedergegeben ist, sei kurz auf die eingangs erwähnten Mitteilungen hingewiesen. In beiden Fällen konnte weder der Beginn noch das Ende des Degenerationsprozesses bestimmt angegeben werden. In Wagenmanns Fall lagen 8 Monate zwischen den beiden Beobachtungen, während Sachsalber seinen Patienten, der unterdessen mit hochgradiger Stauungspapille erblindet war, erst nach $21 / 2$ Jahren wieder $z u$ Gesicht bekam.

In unserem Falle hat sich die Ursache für den Schwund der Markscheiden plötzlich 8 Tage vor der ersten Untersuchung eingestellt und es war die Möglichkeit gegeben, denselben während längerer Zeit mit dem Augenspiegel genau zu verfolgen. Die ischämische Degeneration der Maculagegend war schon sehr weit gediehen, als ungefähr 14 Tage

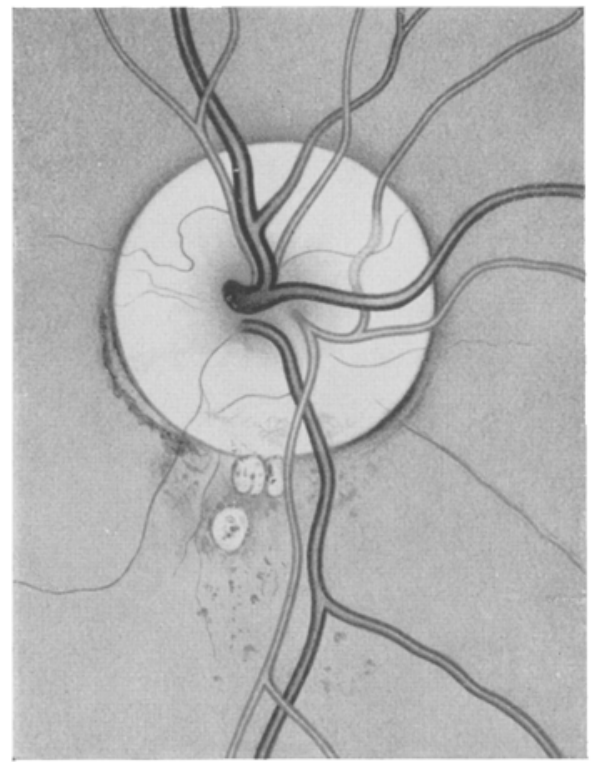

Abb. 3. Befund nach restlosem Schwunde der Nervenfasem.

nach der Embolie die ersten Veränderungen in der Struktur der markhaltigen Fasern wie Auflockerung in feinste weiße Fleckchen unter Verlust des charakteristischen Glanzes wahrgenommen werden konnten. Nach 2 Monaten (Skizze Abb. 1) befand sich schon ein größerer Bezirk nasal unten in vorgeschrittener Auflösung: Bildung von feinen Schollen und Segmenten und glitzernden Einlagerungen; nach weiteren 2 Monaten waren dann schon die mächtigsten Lagen der Fasern, wo die großen Gefäße in ihnen eingebettet waren, soweit rarefiziert, daß der Gefäßlauf durchschimmerte (Skizze Abb. 2). Am schnellsten verlief der Resorptionsvorgang an der Umbiegungsstelle der Nervenfasern am Papillenrand. Bei der letzten Kontrolluntersuchung, 15 Monate nach der Erblindung, konnte man nur mehr von ganz kläglichen Resten der verschwundenen Pracht reden, so daß man nach diesem Zeitraum den Prozeß der ascen- 
dierenden Atrophie als beendet ansehen kann (Skizze Abb.3). Von einer besonderen Reaktion des Zwischengewebes (Wucherung desselben infolge Raumverminderung durch den Schwund der Markscheiden oder durch den Reiz der Degenerationsprodukte) war nichts zu bemerken, auch das Pigmentepithel, das sonst auf Veränderungen in der Nachbarschaft gern reagiert, erwies sich unverändert.

Ein Nebenbefund, der mit Vollendung der Atrophie der Papille in Erscheinung trat, sei noch kurz vermerkt: die feine zilioretinale Arterie in der Papille nasal oben scheint keine echte ,Zilioretinale" zu sein, sondern vielmehr die Fortsetzung eines zuerst verdeckt gewesenen Astes der unteren nasalen Netzhautarterie und also nach Schnabel ein sog. „abirrendes Gefäß $B_{\text {" }}$ darzustellen.

Als Ätiologie des embolischen Verschlusses darf die Mitralinsuffizienz gelten. Es wurde zwar die anamnestisch erhobene Malariainfektion als ursächliches Moment in Erwägung gezogen, aber die genaue, auf der medizinischen Klinik durchgeführte Untersuchung ergab außer dem chronischen Milztumor keinerlei Anhaltspunkte zur Erhärtung einer solchen Annahme: Der Nachweis von Plasmodien und Pigmentresten im Blut gelang auch nach provokatorischen Chiningaben und Milchinjektionen nicht, zudem war Pat. schon über $1 \mathrm{Jahr}$ vollkommen anfallsfrei geblieben, indes die bei Malaria beschriebenen Augenkomplikationen durchwegs bei frischen Erkrankungen oder im Gefolge schwerer anämischer und kachektischer Zustände beobachtet wurden.

Wir haben früher die Atrophie des markhaltigen Nervenbüschels als eine a scendierende bezeichnet, und müssen das noch kurz begründen. Fs ist bekannt, daß das Gewebe des Sehnervenkopfes fast ausschließlich von den kleinen aus dem Zin n - Jägerschen Gefäßkranz in die Papille eintretetenden Gefäßchen ernährt wird. Durch die Embolie der Zentralarterie ist in erster Linie die Gehirnschicht der Netzhaut geschädigt worden und diese anscheinend nicht absolut, da sich in einem Netzhautbezirk innen oben noch in Geringem hierbei Funktionsfähigkeit erhalten, bzw. wieder hergestellt hat. Die markhaltigen Nervenfaserbüschel müssen nicht von der Zentralarterie, sondern vom Ciliargefälssystem ernährt gewesen sein, sonst hätten dieselben das Schicksal der Gehirnschichten der Netzhaut teilen und daher in gleich rapidem Verlaufe der Degeneration und dem Schwund anheimfallen müssen. Der späte Eintritt der Veränderung, der torpide Ablauf der Atrophie dürfte ein Beweis dafür sein, daß die Atrophie der markhaltigen Büschel tatsäohlich als aufsteigende Degeneration, zufolge der Zerstörung der Gehirnschicht der Netzhaut (bzw. der dazu gehörigen Ganglienzellen) zu bezeichnen ist. 